\title{
Macro-instabilities of a Suspension in an Axially Agitated Mixing Tank
}

\author{
M. Jahoda, V. Machoň, L. Vlach, I. Fořt
}

This paper deals with an experimental assessment of the occurrence of flow macro-instabilities in a mechanically stirred suspension and visual observation of the origination and extinction of macro-vortices. The mean frequency of the occurrence of macro-instabilities in operational conditions was also observed. The experiments were carried out in a cylindrical vessel with an inner diameter of $0.19 \mathrm{~m}$, and an axial stirrer with six pitched $45^{\circ}$ blades (PBT) was used. The diameter of the stirrer was equal to half of the vessel diameter. The mean frequencies of the occurrence of macro-instabilities were determined at the stirrer frequency for just suspended conditions in dependence on solids concentration and impeller clearance (height of the stirrer above the bottom of the vessel).

Two regions of origin of macro-instabilities and one region of extinction were determined visually. It was found that the mean frequency of occurrence of macro-instabilities increases with increasing stirrer frequency at constant concentration of solids. At constant stirrer speed, the frequency of occurrence of macro-instabilities decreases with increasing concentration of solids. At higher concentration of solids, a sharp change toward lower mean frequencies of macro-instabilities was observed.

Keywords: suspension, mixing, macro-instabilities.

\section{Introduction}

A planar description of fluid flow caused by a rotational axial impeller in a stirred vessel equipped with radial baffles is usually interpreted by means of a one-loop circulation model. In the case of a pumping-down axial impeller, the flow is directed towards the bottom of the tank. On the bottom, the fluid diverges, changing its direction, and then travels upward along the vessel wall. At the top of the tank, the fluid is directed radially inward and then redirected down to the impeller, closing the circulation loop. However, the real liquid flow inside a tank constitutes a pseudo-stationary high-dimensional dynamical system. Laser Doppler Velocimetry (LDV) and Particle Image Velocimetry (PIV) measurements of the velocity field in stirred vessels revealed the occurrence of a secondary circulation loop near the bottom of the vessel, Kresta and Wood [1] and Myers et al [2]. It was observed

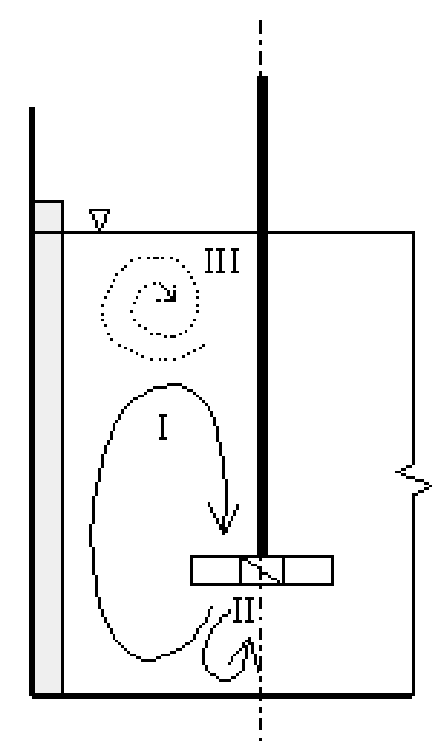

Fig. 1: Flow patterns in a vessel stirred by an axial impeller. Main circulation loop I, secondary circulation loop II, macro-vortex III. further that in the upper third of the vessel the fluid circulation was low and the main circulation loop was instable. Pseudo-periodic flow in the shape of a macro-vortex was detected in this region (see Fig. 1). This phenomenon was also noticeable visually, and it was dependent on stirrer diameter and impeller off-bottom clearance. The measure of these macro-vortices was comparable with the scale of the vessel and this phenomenon was called macro-instability (MI) of the velocity field. Knowledge of the mechanism of the origin and scale of MI is of great practical importance. Macro-vortices improve the mixing in the upper part of the vessel, but they also act negatively on the vessel walls, baffles, impeller shaft, and other solid parts. In addition, the uneven vibration influences the fixation of the apparatus. In extreme cases, it could damage the equipment, Kratěna et al [3].

In the turbulent region of mixing, the presence of macro-vortices in the stirred vessel appears as a swollen surface of the liquid, obviously different from the wavy surface resulting from turbulence eddies. Brůha et al [4] looked at the frequency of MI occurrence according the movement of the liquid surface using 3, 4, and 6 bladed PBT. Their investigations covered two impeller diameters $(D=T / 3$ and $T / 4)$ and three different off-bottom clearances $(T=0.3 \mathrm{~m}, C / T=0.33$, 0.4 and 0.5 ). Based on visual observation, they defined the occurrence of a macro-vortex as a situation during which the liquid surface swells up for more than $5 \mathrm{~mm}$ (under their operational conditions) close to the baffle. They found out experimentally that the occurrence of the macro-vortex is dependent on the number of wall baffles, on the number of stirrer blades and on the stirrer frequency. An electronic probe measured the frequency of macro-vortex occurrence and a linear dependence was found with stirrer frequency. It is not sufficient to look at the liquid surface from the outside to understand the origination of macro-vortices, and vortices that do not reach the surface are not registered.

The authors of the above mentioned study used a mechanical measuring device, developed later, called a "tornadometer", which enabled the frequency of MI inside the vessel to be measured (Brůha et al $[5,6])$. They confirmed their 
previous finding that the mean MI frequency is linearly related to the rotational speed of the impeller. The impeller off-bottom clearance influenced the slope of this dependency. They also observed that the MI frequency is accompanied by changes in the angle of the impeller discharge flow and the appearance of an unstable secondary circulation loop. Further, the dimensionless MI frequency was defined as the mean frequency of MI occurrence divided by the frequency of the stirrer speed, and the dependency of this quantity on modified Reynolds number, Re, was found in three hydrodynamic regimes. In the laminar region of fluid flow in the vessel $(\operatorname{Re}<200)$, the primary circulation loop was steady and the MI was not registered. In the transient region of the flow $(200<\operatorname{Re}<5000)$, the dimensionless frequency of MI was found to increase logarithmically with increasing values of Re. Under fully turbulent conditions ( $\operatorname{Re}>9000)$, the values of MI frequency of occurrence were near to constant in the range from 0.043 to 0.048 . The intensity of the turbulence increased with increasing Re number, and it was not possible to distinguish between MI and turbulent eddies during highly turbulent flow $(\operatorname{Re}>67000)$. Generally, the measuring method with a tornadometer is regarded by many authors as unreliable, because of the presence of the arm inside the vessel, which could influence the fluid flow.

Montes et al [7] use LDV measurements and spectral analysis to observe MI frequency occurrence in a stirred tank equipped with a six-bladed $45^{\circ}$ PBT impeller $(T=0.3 \mathrm{~m}$, $D=T / 3, C / T=0.35)$. The axial and radial components of the velocity were measured at eight locations situated evenly in a rectangular net in the stirred region. The measurements lasted 25 minutes and the time record of the velocity was analysed by the Fast Fourier Transform (FFT). The dominant frequency was determined from the frequency spectrum. The dominant frequencies were proved in all measurement locations, but the obtained values of the power spectrum density were not identical. It was concluded that MI are macroscopic phenomena with a dimension comparable with the dimension of the apparatus. The results confirmed the previous finding that the mean frequency of MI increases linearly with the stirred frequency.

Hasal and Fořt [8] applied a non-linear dynamic system analysis method to data measured by Montes et al [7]. They demonstrated the possibility to detect and separate the low dimensional component of the velocity field from the stochastic turbulent component. In the velocity field, the macro-instabilities presented a marked component of kinetic energy. The presence of MI in the velocity record was more distinct in laminar and transient flow regimes. The macro-instabilities were poorly manifested in the discharge flow of the impeller, where their relative magnitude takes on a minimal value. The authors deduced possible production of MI from the interaction of the impeller discharge stream and the ascending circulation streams.

Roussinova and Kresta [9] analysed the LDV time series of axial velocities upstream of the baffle using the Lomb algorithm as an alternative method to FFT for the case of unevenly spaced data. They found a dominant MI frequency of $0.62 \mathrm{~Hz}$ in a system stirred by a pitched blade $45^{\circ}$ turbine (4 blades) with diameter $D=T / 2 \quad(T=0.24 \mathrm{~m})$ and $\operatorname{Re}=48 \cdot 10^{4}$. The impeller off-bottom clearance $(C / T=0.33$, 0.5 , and 0.67 ) did not change the value of the dominant frequency. The dimensionless MI frequency was constant at 0.18 for $\operatorname{Re}>10^{4}$. No dominant frequency appeared for small PBT $(D=T / 4)$ at any impeller off-bottom clearance.

The previous investigations show that the incidence of macro-instability is very sensitive to vessel geometry and the procedure of MI frequency determination. Our goal was to examine experimentally the occurrence of macro-instabilities in a mechanically stirred system in which the solid particles were present and fully suspended.

\section{Experimental}

The experiments were carried out in a stirred cylindrical baffled vessel with a flat bottom and inner diameter $T=0.19 \mathrm{~m}$. A sketch of the experimental tank is shown in Fig. 2. The mixed charge was a suspension in water of solid particles at room temperature, and the vessel was filled to the height $H=T$. An outer square glass tank was filled with water in order to minimize the effects of vessel curvature on visual observation. The axial impeller was a pitched blade turbine (PBT) of diameter $D=T / 2$ with six blades inclined at $45^{\circ}$ (pumping down), and its off-bottom clearances were $C / T=0.2,0.3$, and 0.4. The width of the blade, $h$, was one fifth of the impeller diameter $D$. The impeller speed, $N$, was varied in the range from $75 \mathrm{rpm}$ to $200 \mathrm{rpm}$. The PVC solid particles were spherical in shape, diameter $0.94 \mathrm{~mm}$, and density $1260 \mathrm{~kg} \mathrm{~m}^{-3}$. The solids concentration was changed from $2.5 \%$ to $22.5 \%$ by weight (from $2 \%$ to $18.7 \%$ by volume). The experiments were done in conditions where the solid particles were fully suspended or in a condition near to full suspension. The visual method, Zwietering [10], was used for assessment of the minimal impeller speed at which the solid particles were just suspended, $N_{\mathrm{js}}$.

To investigate MI frequency occurrence, the upper part of the mixing vessel close to the baffle leading edge was observed (see Fig. 2). The presence of red colored solid particles made it possible to observe the appearance of macro-vortices. A typical spiral shape characterized a fully developed macro-vortex. A video camera was used to reduce the spatial view for visual observation. The time between two successive appearances of macro-vortices was stored in the computer memory in accordance with a signal from an

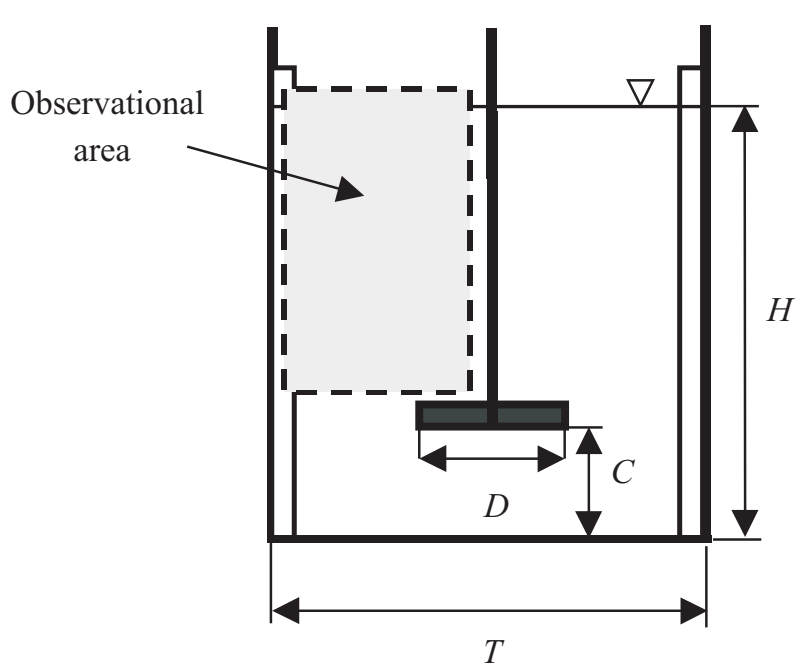

Fig. 2: Experimental vessel 
operator. For each experimental condition, the recording time was about $30 \mathrm{~min}$. The reciprocal values of the time series give the incidence of MI. The mean values of MI frequency occurrence, $f_{\mathrm{M}}$, and its dimensionless form, $f_{\mathrm{MI}}=f_{\mathrm{M}} / N$, were then calculated.

\section{Results and discussion}

During the experiments, two circulation flow patterns were observed. There was a stable and symmetrical circulation pattern around impeller speed $65 \mathrm{rpm}(C / T=0.2,0.3$, and 0.4 ), which resembled the flow described by Kresta and Wood [1]. The single primary circulation loop reaches a height of only $2 / 3 H$. This is in agreement with the results presented by Bittorf and Kresta [11]. If the impeller speed increased, the stable flow pattern turned into unstable conditions. The primary circulation loop is unstable and macro flow instabilities begin to appear. An upper part of the mixed vessel was stirred mainly by means of the macro-vortices. These vortex structures are able to reach the free surface if they have sufficient energy. A local swell due to a macro-vortex was apparent for impeller speed around $200 \mathrm{rpm}$. This corresponds to the description made by Brůha et al [4].

Two regions of origin of macro-instabilities and one region of extinction were determined visually. The first area of origin was along the leading edge of the baffle. A stream moving up beside a baffle usually headed to the impeller area, but sometimes the flow was accelerated and reached the free surface or came near to the surface. Macro-vortices developed from this jet stream (see Fig. 3). Similar macro-vortex creation was observed by Montes et al [7]. The second area of origin was located between adjacent baffles at a horizontal distance $0.25 \mathrm{~T}$ from vessel wall to shaft (see Fig. 3). The macro-vortices were formed on an interface between the ascending stream of suspension along the vessel wall and the descending stream that arrived from the baffle trailing edge. The stream interface was nearly constant in the vertical direction from the vessel bottom (from $0.45 T$ to $0.5 T$ ) for impeller speed above $N_{\text {js }}$ and for all investigated off-bottom impeller clearances. The horizontal position of the interface was slightly pitched due to stream that arrived from the baffle trailing edge. The area of macro-vortex extinction was always in the upper quadrant of the stirred vessel near the baffle leading edge and the free surface, and it was not dependent on the macro-vortex origin. The frequency of macro-vortex occurrence was in the range from 0.5 to $2 \mathrm{~s}^{-1}$.

The dependence of the mean value of the dimensionless frequency of macro-instability occurrence on impeller speed for constant solid concentration $10 \%$ by weight is shown in Fig. 4. The figure exposes some influence of the amount of solid particles in suspension on macro-vortex detection. The minimal impeller speed for full suspension of solid particles, determined by Zwietering's method, was found to be $135 \mathrm{rpm}$. This method is very dependent on the opinion of an observer. It seems that the real value of $N_{\mathrm{js}}$ ranged from 135 to $150 \mathrm{rpm}$. For higher impeller speed, the solid particles were fully suspended and the dimensionless frequency values of MI are practically steady. This behaviour conforms with a one-phase system in which the dimensionless MI frequency scarcely changes in the region of developed turbulence.
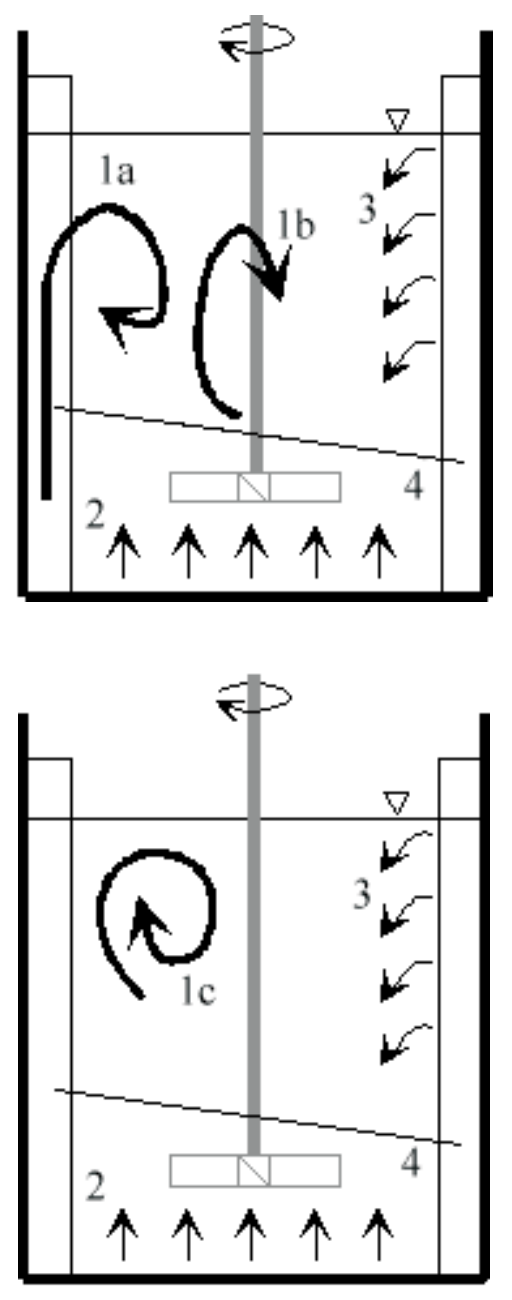

Fig. 3: Sketch of origin of macro-instabilities.

1a) The area of origin was along the leading edge of the baffle.

1b) Area of origin between two adjacent baffles.

1c) Fully developed macro-vortex.

2) Ascending stream along the vessel wall.

3) Descending stream arriving from the baffle trailing edge.

4) Interface between ascending and descending streams.

The dependence of the mean value of the dimensionless frequency of macro-instability occurrence on solid concentration for constant impeller speed is shown in Fig. 5. The solid particles were in the fully suspended state. There is

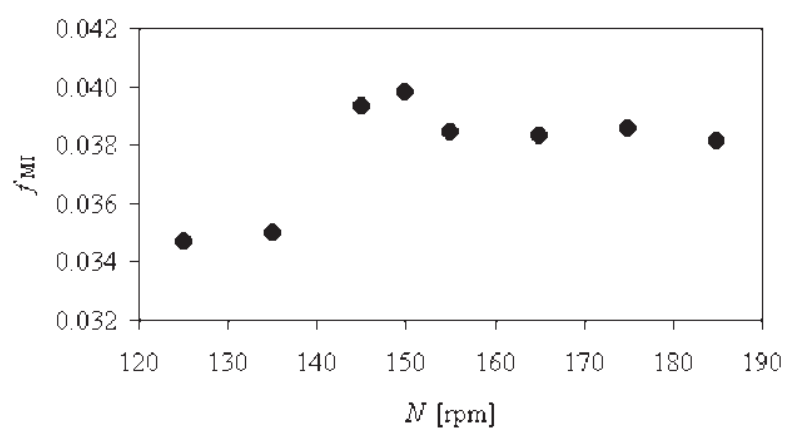

Fig. 4: Dependence of mean value of dimensionless MI frequency on impeller speed for $C=0.2 T, N_{\mathrm{js}}=135 \mathrm{rpm}$, $w=10 \% \mathrm{w} / \mathrm{w}, 1.4 \cdot 10^{4}<\operatorname{Re}<2.8 \cdot 10^{4}$ 


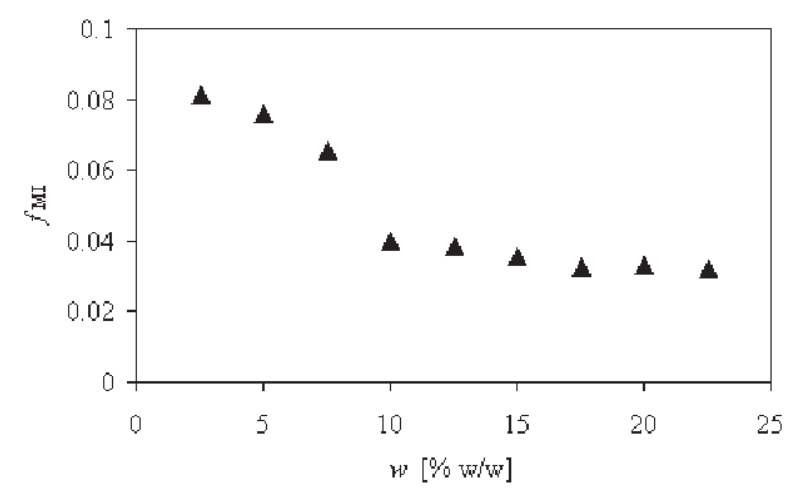

Fig. 5: Dependence of the mean value of dimensionless MI frequency on solid concentration for $C=0.2 T$ and $N=150 \mathrm{rpm}$

a turning point between solids concentrations $7.5 \% \mathrm{w} / \mathrm{w}$ and $10 \% \mathrm{w} / \mathrm{w}$. Solid particles above a concentration of $7.5 \% \mathrm{w} / \mathrm{w}$ greatly influenced the fluid flow inside the vessel. From visual observation, it was apparent that the flow patterns were more stable in the case of higher solids concentrations than at lower concentrations.

Figure 6 depicts the dependence of the mean value of the dimensionless frequency of macro-instability occurrence on solid concentration for minimal impeller speed when the solid particles were just suspended. The values of

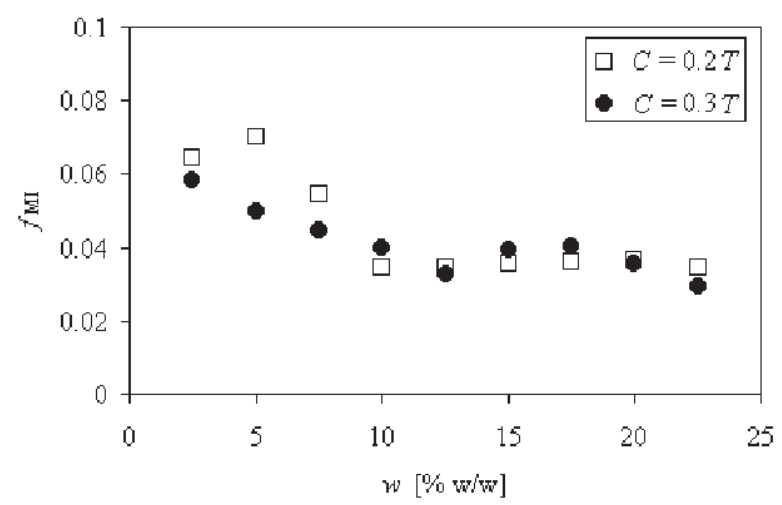

Fig. 6: Dependence of the mean value of dimensionless MI frequency on solid concentration for $N_{\mathrm{j} s}$

dimensionless MI frequency were very similar for the two off-bottom clearances, $C=0.2 T$ and $0.3 T$, above all when the solids concentration was higher than $7.5 \% \mathrm{w} / \mathrm{w}$. In the case of impeller off-bottom clearance $0.4 T$ and $w>15 \% \mathrm{w} / \mathrm{w}$, the investigation of macro-vortex incidence could not be carried out due to a high degree of turbulent eddies.

\section{Conclusions}

The existence of macro-instability occurrence in the examined mixing system was proved by visual observation for a wide range of solid particles concentrations and three off-bottom impeller clearances. Two regions of origin of macro-instabilities and one region of extinction were determined. The macro-vortices evolved either in an area along the leading edge of the baffle or at a location between the baffles at a horizontal distance $0.25 \mathrm{~T}$ from vessel wall to impeller shaft. The macro-vortices always disappeared in the upper quadrant of the stirred vessel near the baffle leading edge and free surface. The frequency of macro-vortex occurrence was found to be in the range from 0.5 to $2 \mathrm{~s}^{-1}$. The visual method is very simple but demanding on the attentiveness of the operator. The mean value of the macro-instability frequency is linearly related to the impeller rotational speed for equal concentrations of solids particles. The mean value of the dimensionless frequency of macro-instability occurrence is steady above the minimal impeller speed for just suspension conditions. At constant stirrer speed, the frequency of occurrence of macro-instabilities decreases with increasing concentration of solids. A turning point was observed between solids concentrations $7.5 \% \mathrm{w} / \mathrm{w}$ and $10 \% \mathrm{w} / \mathrm{w}$. At higher concentration of solids, the mean frequencies of the macro-instabilities were identical. The results for impeller off-bottom clearances $0.2 T$ and $0.3 T$ are very similar.

\section{List of Symbols}

C impeller off-bottom clearance, $\mathrm{m}$

$D \quad$ impeller diameter, $\mathrm{m}$

$f_{\mathrm{M}}$ mean value of frequency of occurrence of macro-instabilities, $\mathrm{s}^{-1}$

$f_{\text {MI }}$ mean value of dimensionless frequency of occurrence of macro-instabilities

$H \quad$ height of suspension in the vessel, $\mathrm{m}$

$h \quad$ width of impeller blade, $m$

$N \quad$ impeller speed, $\mathrm{s}^{-1}$

$N_{\text {js }} \quad$ minimal impeller speed for just suspended condition, $\mathrm{s}^{-1}$

Re Reynolds number

$T$ vessel diameter, $\mathrm{m}$

$w \quad$ solid particles concentration by weight

\section{Acknowledgement}

This research was financially supported by Research Projects of the Ministry of Education of the Czech Republic J19/98:223400007 and J04/98:2122 0008 .

\section{References}

[1] Kresta, S. M., Wood, P. E.: The mean flow field produced by a $45^{\circ}$ pitched blade turbine: changes in the circulation pattern due to off bottom clearance. Canad. J. Chem. Eng., 1993, 71, p. 42-53.

[2] Myers, K. J., Ward R. W., Bakker A.: A digital particle image velocimetry invesigation of flow field instabilities of axial-flow impellers. J. Fluids Eng., 1997, 119, p. 623-632.

[3] Kratěna, J., Fořt, I., Brůha, O., Růžička, M.: Dynamic stress affecting the radial baffle in an industrial mixing vessel with a pitched blade impeller. Acta Polytechnica, 2000, Vol. 40, No. 5/6, p. 22-31.

[4] Brůha, O., Fořt, I., Smolka, P.: A large scale unsteady phenomenon in a mixing vessel. Acta Polytechnica, 1993, Vol. 33, No. 4, p. 27-34. 
[5] Brůha, O., Fořt, I., Smolka, P.: Phenomenon of turbulent macroinstabilities in agitated systems. Collect. Czech. Chem. Commun. 1995, Vol. 60, p. 85-96.

[6] Brůha, O., Fořt, I., Smolka, P., Jahoda, M.: Experimental study of turbulent macroinstabilities in an agitated system with axial high - speed impeller and with radial baffles. Collect. Czech. Chem. Commun., 1996, Vol. 61, p. 856-867.

[7] Montes, J. L., Boisson, H. C., Fořt, I., Jahoda, M.: Velocity field macro-instabilities in an axially agitated mixing vessel. Chem. Eng. J., 1997, Vol. 67, p. 139-145.

[8] Hasal, P., Fořt, I.: Macro-instabilities of velocity field in stirred vessel: detection and analysis. Chem. Eng. Sci. 2000, Vol. 55, p. 391-401.

[9] Roussinova, V., Kresta, S. M.: Analysis of macro-instabilities of the flow field in a stirred tank agitated with axial impellers. $10^{\text {th }}$ European Conference on Mixing, Delft, The Netherlands, 2000, p. 361-368.

[10] Zwietering, T. N.: Suspending solid particles in liquids by mechanical mixers. AICHE J., 1958, Vol. 6, No. 3, p. $419-426$.
[11] Bittorf, K.-J., Kresta, S. M.: Active volume of mean circulation for stirred tanks agitated with axial impellers. Chem. Eng. Sci., 2000, Vol. 55, p. 1325-1335.

Dr. Ing. Milan Jahoda

phone: +420224353223

e-mail: Milan.Jahoda@vscht.cz

Doc. Ing. Václav Machoň, CSc.

Ing. Lubomír Vlach

Dept. of Chemical Engineering

Prague Inst. of Chemical Technology

Faculty of Chemical Engineering

Technická 3, 16628 Praha 6, Czech Republic

Doc. Ing. Ivan Fořt, DrSc.

phone: + 420224352713

Dept. of Process Engineering

Czech Technical University in Prague

Faculty of Mechanical Engineering

Technická 4, 16607 Praha 6, Czech Republic 